\title{
MRI of anterior spinal artery syndrome
}

\author{
Akito Kume, Sakae Yoneyama, Akira Takahashi, Hideo Watanabe
}

\begin{abstract}
In this serial MRI study a 24 year old man presenting anterior spinal artery syndrome was examined. In the acute stage, spin echo sequences showed an enlarged cervical cord on a $T 1$ weighted image and high signal intensity in the enlarged portion of the cord on a T2 weighted image. The findings were interpreted as oedema in the grey and white matter subsequent to ischaemia. In the chronic stage, inversion recovery techiques revealed a distinct focus in the anterior two thirds of the cord at the low cervical level.
\end{abstract}

( Neurol Neurosurg Psychiatry 1992;55:838-840)

Anterior spinal artery syndrome (ASAS) ${ }^{12}$ is a neurological syndrome of spinal cord infarction caused by an occlusion of the spinal part of the anterior spinal artery. This vessel supplies about two thirds of the cross-sectional area of the cord, but not the posterior part of the posterior white columns and the posterior grey horns. The onset is abrupt, with pain, flaccid paraplegia or tetraplegia below the lesion, and impairment of heat and pain but not deep sensation. We present a case of sequential MRI study confirming a spinal cord lesion consistent with ASAS.

\section{Case report}

A 24 year old mechanic woke up because he felt ill. He flexed his neck while urinating, an aching of the neck developed followed by muscular weakness in both legs. He had no history of a previous trauma or concurrent infection. Three hours later the weakness extended to the arms and he was transferred to our hospital. On arrival, he could walk without support and felt a constant urge to urinate. More than eight hours after admission, the weakness was steadily progressing and urinary retention developed. The next morning, the patient was alert and fully conscious, complaining of slight respiratory distress. His cranial nerve functions were normal but he could not move his head satisfactorily, and there was complete flaccid tetraplegia. Superficial and deep reflexes were absent below the cervical level. All sensory modalities from C5 to T3 were impaired, and there was an aesthesioanalgesia below T4. The physical examination revealed no abnormalities: body temperature was $36.6^{\circ} \mathrm{C}$, blood pressure
$98 / 56 \mathrm{~mm} \mathrm{Hg}$, and a regular pulse of 78 beats a minute.

Routine laboratory examinations were unremarkable. $X$ rays of the skull, cervical spine and chest were normal. CSF pressure was $80 \mathrm{mmH}_{2} \mathrm{O}$, jugular compression test was normal, and the CSF was clear and colourless. The CSF protein content was $0.67 \mathrm{~g} / 1$ on the second day and 0.28 on the twentieth day. Other CSF examinations, including cell count, IgG, myelin basic protein, and cytology were all normal.

A myelogram showed an enlargement of the cervical cord. High resolution CT did not disclose any haematoma in the cord. Electromyography revealed anterior horn cell damages on $\mathrm{C6}$ and $\mathrm{C7}$, and somatosensory evoked potentials in the arms and legs demonstrated a conduction block above C7. MRI findings are described further below.

The neurological deficit stabilised after the first 12 hours. The patient was treated with daily intravenous methylprednisolone, $1000 \mathrm{mg}$ for three days and oral prednisolone $60 \mathrm{mg}$ for four weeks. After the first week he gradually began moving his arms and hands again. Two weeks later marked spasticity, hyperreflexia of the legs and positive Babinski's signs appeared. While deep sensation returned to normal, the loss of pain and thermal sensation persisted below T4 ("dissociated sensory impairment"). Four weeks later atrophy of the small hand muscles became apparent. Muscle power of the deltoid, biceps and brachioradial muscles eventually returned to more than $50 \%$ of the normal measure, but recovery of the triceps muscle of the arms and distal muscles was limited to less than $25 \%$, while the legs remained completely paraplegic. Urinary retention persisted and self catheterisation was required. The neurological findings did not change during the following 12 months and the patient became confined to a wheelchair. There has been neither progression nor recurrence for the last three years.

\section{MRI}

MRI was performed on a Picker 0.5 tesla imager using the multislice, spin echo (SE) technique for $T 1$ weighted $(T R=500 \mathrm{~ms}$, $\mathrm{TE}=40 \mathrm{~ms})$ and $\mathrm{T} 2$ weighted (TR = $1000 \mathrm{~ms}, \mathrm{TE}=120 \mathrm{~ms}$ ) sequences and the inversion recovery (IR) technique for $T 1$ weighted sequence $(T R=1500 \mathrm{~ms}, T I=$ $500 \mathrm{~ms}$ ). We produced sagittal images with 
Figure Forty eight hours after onset of illness, the T2 weighted image ( $S E$ scan) displays high signal intensity in the enlarged portion of the cord $(A$, arrowheads). After two months of illness, the IR scan demonstrates a distinct focus in the anterior two thirds of the cord at the low cervical level and the sparing of only the posterior one third (B, arrows).

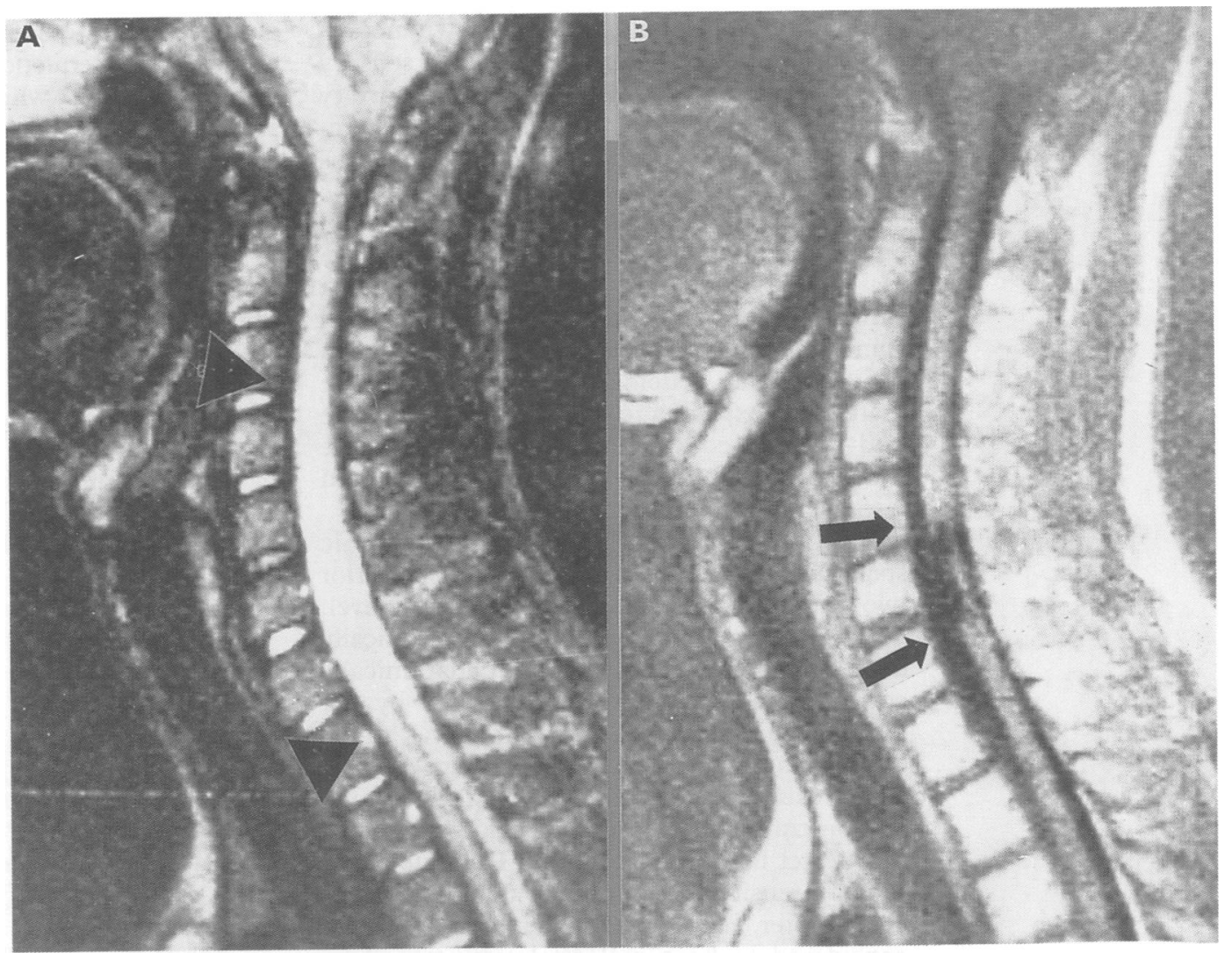

$5 \mathrm{~mm}$ contiguous slices and a field of view of $30 \mathrm{~cm}$ (see table).

The initial MRI obtained 48 hours after the onset of the illness (see table and fig) showed an enlargement of the cervical cord on T1 weighted images ( $\mathrm{SE}_{500 / 40}$ and IR scans), with a diffuse, abnormal increase in signal intensity over four levels in the substance of the cord on T2 weighted images ( $\mathrm{SE}_{1000 / 120}$ scans). A follow up examination two months later showed that the size of the cord had returned to normal and that the range of high signal intensity on $\mathrm{T} 2$ weighted images $\left(\mathrm{SE}_{100 / 120}\right.$ scans) was reduced to one or two segments. This region of high signal intensity was too poorly defined to outline the boundaries of the lesion exactly. IR scans, however, revealed a distinct focus in the anterior two thirds of the cross-sectional area at the low cervical level around $\mathrm{C} 7$ and the sparing of the posterior one third of the spinal cord that corresponds to the posterior column. The focus was much darker than the grey matter and as black as CSF, in strong contrast to the substance of the cord. There was no abnormal signal suggesting a haematoma. There were almost identical findings in the third MRI study carried out in the seventh month of his illness.

\section{Discussion}

We believe the patient suffered from an infarction of the spinal cord because of the typical clinical features, absence of cord compres-

Table Clinical and MRI correlation in the patient with anterior spinal artery syndrome

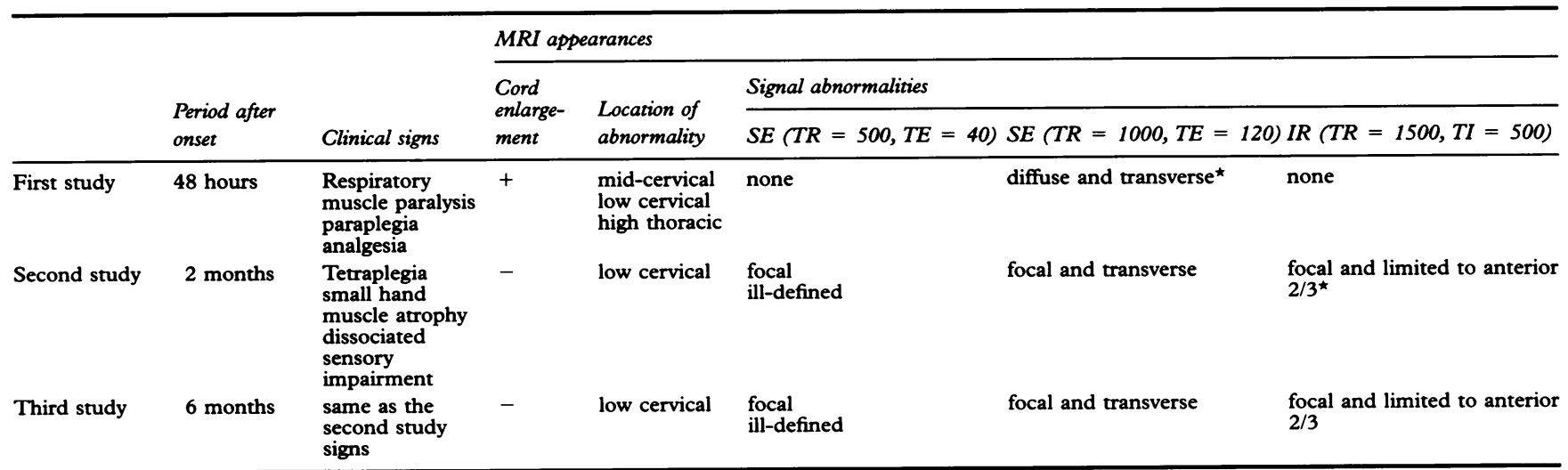


sions, and exclusion of other known neurological disease. ${ }^{23}$ A typical dissociated sensory impairment pointed to anterior spinal artery syndrome (ASAS) ${ }^{12}$ as the result of an infarction occurring in the region supplied by this artery.

MRI during the acute stage showed an enlargement of the cervical cord and an increased T2 signal in the enlarged portion. A similar finding was reported in patients with spinal cord infarction or ischaemia, ${ }^{4-6}$ and a post-ischaemic oedema in the grey and white matter was expected to occur. ${ }^{5}$ In our case, the mid-cervical cord oedema may explain the transient respiratory muscle paralysis and tetraplegia observed during the acute stage.

It is noteworthy that IR scans in the chronic stage disclosed a distinct focus in the anterior two thirds of the cord at the low cervical level. This location coincided with the clinically determined site of the leson and anatomically corresponded to the region supplied by the anterior spinal artery. ${ }^{2}$ The signal was much lower than that of the grey matter. A MRI study using a IR sequences similar to ours demonstrated a well defined region of a chronic infarct in CNS with a long T1 value and a signal intensity that was lower than that of the grey matter. ${ }^{7}$ The focus in our case therefore considered to be a malacic lesion caused by anterior spinal artery infarction.

Although there are several reports on ASAS being depicted by $\mathrm{MRI},{ }^{8-10}$ MRI cannot always detect the lesion of ASAS. ${ }^{11}$ The clinical application of IR sequences has proven to be quite valuable to locating brain lesions. ${ }^{12}$ In our case, IR sequences provided satisfactory imaging of ASAS, while SE scans did not yield a good enough contrast between the substance of the cord and the lesion of infarct.

We are grateful to Dr T Kato and Dr H Asai for radiological assistance and Dr T Andoh for his helpful suggestions.

1 Preobrashenski PA. Syphilitic paraplegia with dissociated disturbance of sensation. Zh Nevropatol Psikhiatr 1904; 4:388.

2 Lazorthes G. Pathology, classification and clinical aspects of vascular diseases of the spinal cord. In: Vinken PJ, Bruyn GW, eds. Handbook of clinical neurology, vol 12. AmsterGW, eds. Handbook of clinical neurology
dam: North Holland, 1972:492-506.

3 Bermann M, Feldman S, Alter M, Zilber N, Kahana E Acute transverse myelitis: incidence and etiologic considerations. Neurology 1981;31:966-71.

4 Brown E, Virapongse C, Gregorios JB. MR imaging of cervical spinal cord infarction. F Comput Assist Tomogr 1989;13:920-2.

5 Mawad ME, Rivera V, Crawford S, Ramirez A, Breitbach W. Spinal cord ischemia after resection of thoracoabdomina aortic aneurysms: MR findings in 24 patients. AfNR 1990;11:987-91.

6 Mark AS. Non-degenerative, non-neoplastic diseases of the spine and spinal cord. In: Atlas SW, eds. Magnetic resonance imaging of the brain and spine. New York: Raven Press, 1991:967-1011.

7 Bydder GM, Steiner RE, Young IR, Hall AS, Thomas DJ, Marshall J, Pallis CA, Legg NJ. Clinical NMR imaging of the brain: 140 cases. $A \mathcal{H} R$ 1982;139:215-36.

8 Inoue $K$. MRI of anterior spinal artery syndrome. fpn $\mathcal{f}$ Stroke 1988;10:517-22.

9 Uyama E, Uchino M, Yamanaka N, Araki S. MRI findings of anterior spinal artery syndrome on chronic stage. Neurol Med (Tokyo) 1989;30:633-5.

10 Amoiridis G, Poehlau D, Przuntek H. Neurophysiological findings and MRI in anterior spinal artery syndrome of the lower cervical cord: the value of F-waves. $\mathcal{F}$ Neurol Neurosurg Psychiatry 1991;54:738-40.

11 Markusse HM, Haan J, Tan WD, Breedveld FC. Anterior spinal artery syndrome in systemic lupus erythematosus. Br ₹ Rheumatol 1989;28:344-6.

12 Bydder GM, Young IR. MR imaging: clinical use of the inversion recovery sequence. $\mathcal{F}$ Comput Assist Tomogr 1985;9:659-75. 